\title{
Design of Real-time Data Acquisition System for Physical Education in Elementary and Secondary School
}

\author{
X.Y. Zhang, X.M. Ju \\ Software Eng ineering Institute \\ East China Normal University \\ China
}

\begin{abstract}
At present, the physical education in elementary and secondary school lacks real-time data acquisition system, which resulted in obstacles to relevant researches. In this paper, a realtime sports data acquisition system for multiple students is proposed. Combined with wireless communication technology, the paper designs and develops a sports data collector and wireless base station for receiving data. The process of data communication utilizes MINA frame. Also, a complete set of Socket-based data transfer protocols is defined. The system not only provides researchers with reliable data, but also enables PE teachers to monitor students' physical exercise in real time. Through the computer stimulation test and field test, both the performance and the availability of the system are verified.
\end{abstract}

Keywords-real-time data acquisition; physical education; Socket; MINA

\section{INTRODUCTION}

With the development of technology, wearable device came into being [1]. Simply speaking, a wearable device is the computer that can be worn on human body and meets people's different computing needs [2]. Under this circumstance, the product that combines wearable computers with sports activities becomes a current hot spot. Some international sporting goods companies have already published such products targeting at exercisers and sports fans. The principle of these products is using sensors to record exercise data, then the data are sent to users' smart phone or network server by Bluetooth or other wireless ways, after which the data are presented on mobile application or accompanying website. However, the existing products are aimed at single user only, and unable to collect data from multiple users [3][4]. Besides, only at the end of sports activities can users access the data. In addition, the distance between the smart phone and sensors on users' body is usually not more than 10 meters [5]. It is so close that the communication range cannot cover one standard playground in elementary and secondary school. And these expensive commercial products are hard to popularize among teenagers because of high price.

In order to deal with these problems, this paper designs a real-time data acquisition system for physical education in elementary and secondary school, develops a sports data collector and wire less base station to transmit data, and defines a set of Socket-based data transfer protocol. The project relies on Adolescent Health Assessment and Exercise Interference Key Laboratory of Education Ministry in East China Normal
University, and the goal is to monitor teenagers' exercise in school, and offers data to reliable scientific researchers. In the following part, this paper will present system's framework, communication method and test results.

\section{DAT A ACQUISIT ION SYSTEM STRUCTURE}

The data acquisition system designed in the paper includes three major parts: wearable data collector, wireless base station, and base station server. Meanwhile, we develop an accompanying Android mobile terminal and an online service website. Users are able to get data fro $m$ the diagrams or tables shown on these two platforms. The structure of the system is shown in Figure1.

The wearable data collector is composed of $3 \mathrm{D}$ accelero meter, MCU micro controller, lithium battery, power management system, data storage module, and ultra-low power wireless communication module. The data collector is compact and portable in appearance, and can be worn at the waist. The main function of data collector is to record the number of steps during exercise. Also, it sends data to base station through $2.4 \mathrm{GHz}$ wireless network.

Base station consists of ultra-low power RF communication module, data storage module, high-performance MPU microprocessor, and network controller. Base station acts as a transfer stop for real-time data. It stores students' sports data temporarily during PE classes, and sends the data to Android mobile terminal through base station server. In this way, teachers are able to monitor exercise situation in real time.

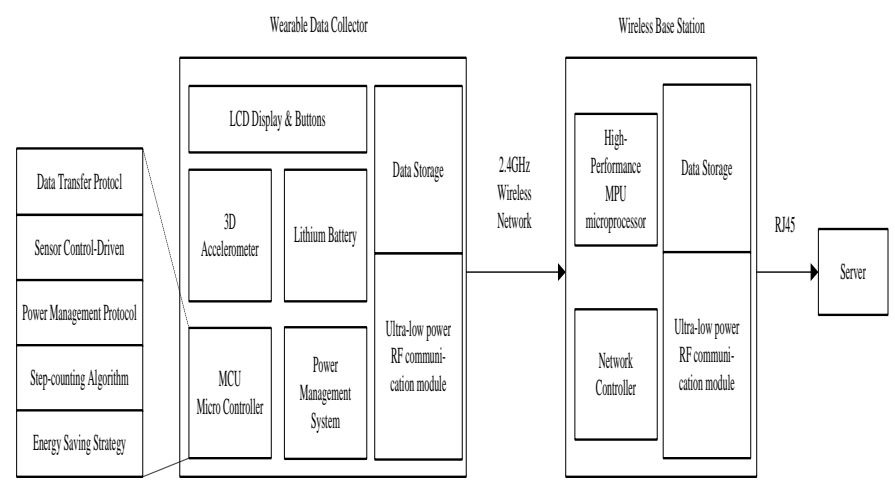

FIGURE I. DAT A ACQUISIT ION SYSTEM ST RUCTURE. 


\section{DAT A COMMUNICATION}

Data acquisition system determines the accuracy of sports data directly. Particu larly, real-time data acquisition system for physical education in elementary and secondary school puts forward more requirements for data communication.

\section{A. Wireless Communication Standards}

In the project, the communication range between wearable data collectors and wireless base station should cover a standard playground which contains 400-meter runway. Firstly, three common wireless communication standards widely used in current products are compared and analyzed: Bluetooth, Wi$\mathrm{Fi}$, and Zigbee [6].

Bluetooth does not satisfy the requirements because of short communication distance [7]. Though the maximum theoretical communication distance of Zigbee meets the requirements, the data rate of Zigbee is far lower than that of Wi-Fi [8]. By contrast, Wi-Fi is widely used in modern life [9], through which the networking process can be achieved conveniently. Therefore, Wi-Fi is the most suitable technology for wireless communication in the project.

\section{B. Communication Networking}

When the class begins, an ad-hoc sensor network is built among data collectors and base station automatically. In this system, there is no communication among different data collectors. Data collectors merely communicate with wireless base station, receiving the instructions from base station and sending feedback. On this occasion, data collectors are only responsible for recording students' exercise data. Hence, the wireless network adopts star topology [10]. It is easier to maintain in elementary and secondary school.

While building wireless network, firstly, the base station sweeps to set up working platform. After receiving instructions, each data collector broadcasts its own MAC address, and obtains communication channel from base station. Then, the base station sends an ACK message to complete the process of establishing the communication channel. Inside the base station, a node list is maintained. The base station calls the LISTEN event in Socket continuously to check whether a message is sent by data collectors or not. AES encryption algorith $\mathrm{m}$ is adopted in the process data transfer.

\section{Base Station Server}

The communication servers in the whole system include two parts: base station server and Android communication server. In this paper, we focus on base station only. The communication between wire less base station and base station server, which is maintained by heartbeat, applies Socket communication mechanism and keep-alive connection.

The structure of base station server is demonstrated in Figure 2. In order to provide high-performance asynchronous IO services, the Socket communication employs Apache MINA framework (IOService and IOFilterChain in Figure 2). Since a base station usually serves multiple data collectors, this paper designs a multi-level thread structure to ensure high concurrency. The design of thread pool is shown in Figure 3.

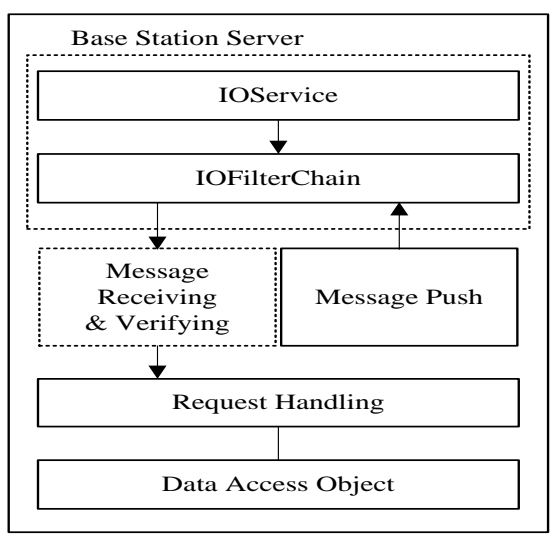

FIGURE II. BASE ST ATION SERVER.

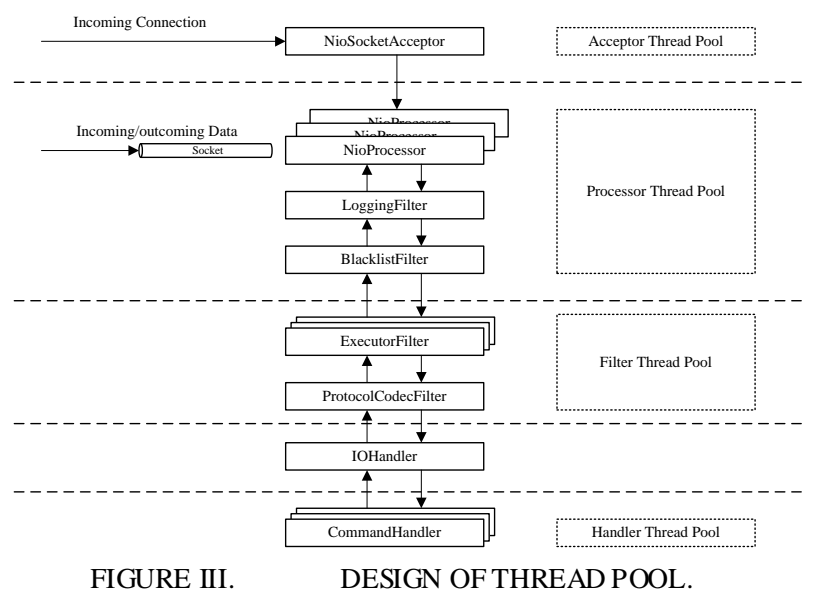

NioAcceptor thread pool builds a corresponding thread for each listening address to listen to client request. Once the connection is successfully established, threads in NioAcceptor pool will handle the client request. The threads in NioAcceptor thread pool handle $\mathrm{I} / \mathrm{O}$ operation. Filter processing chain is also contained in this kind of thread. To process multi-user data in real time, ExecutorFilter thread pool separates timeconsuming filter processing and IOHandler from NioProces sor, which enables NioProcessor to manage more requests. The design of CommanHandler thread pool is based on the producer-consumer model. When IOHandler obtains request from the rear of Filter processing chain and sends the request to CommandHandler thread pool, the thread in CommandHandler pool will manage the real service logic processing.

\section{Data Protocol}

For the communication between wearable data collectors and base station, a complete set of data transfer protocol is defined. The data packet format is shown in Table 1. The protocols are divided into two types: for the instructions sent by base station and replied by data collectors, and for the instructions without replies from data collectors. The former type includes protocols to obtain data collector ID, get battery information of data collectors, to acquire the number of steps, set current time for data collectors, set exercise amount index for data collectors, and reset data collectors, 6 items in total. 
The later type includes protocols for data collectors to broadcast addresses, for base station to modify data collectors' addresses, to confirm the survival of base station, and for data collector to get auto-answer, 4 items in total. Protocols to obtain data collector ID, acquire the number of steps, and set exerc ise amount index for data collectors are closely related to exercise data acquisition.

\section{TABLE I. DATA PACKET FORMAT.}

\begin{tabular}{|c|c|c|c|c|}
\hline & $\begin{array}{c}\text { Instruction } \\
\text { Code }\end{array}$ & Serial Number & Data & Identifier \\
\hline Length & 2 bytes & 2 bytes & N bytes & 2 bytes \\
\hline Type & BYTE & BYTE & BYTE & BYTE \\
\hline
\end{tabular}

\section{TEST RESULTS}

The purpose of this test is to validate the system's processing capacity, and verify concurrent handling ability of Apache MINA. The test program creates $n$ threads, and each thread sets up a connection with the server. Every connection continuously sends $\mathrm{m}$ requests. According to the project requirements, response latency is limited to 3 seconds. In this test, each request sends 27 bytes of data, and receives 35 bytes of data. After all the connections are established, the timer starts and all the connections send requests simultaneously. The timer stops when all the requests are sent. Here, $m=2000$. The result of computer simu lation test is shown in Figure 4.

From Figure 4, we clearly learn that, with the increase of the number of threads, the processing time decreases sharply, proving the performance of the design, which satisfies the design requirement.

Apart from computer stimulation test, we conducted field test (shown in Figure 5) as well. We deployed the data acquisition system outdoor on the playground. The site is about 174 meters in length and 95 meters in width. In the test, 30 people wore data collectors on the bodies and did physical exercise for 40 minutes. The data collectors record the nu mber of steps of testers and send the data to base station. Then, the data can be displayed on Android mobile terminal in real time.

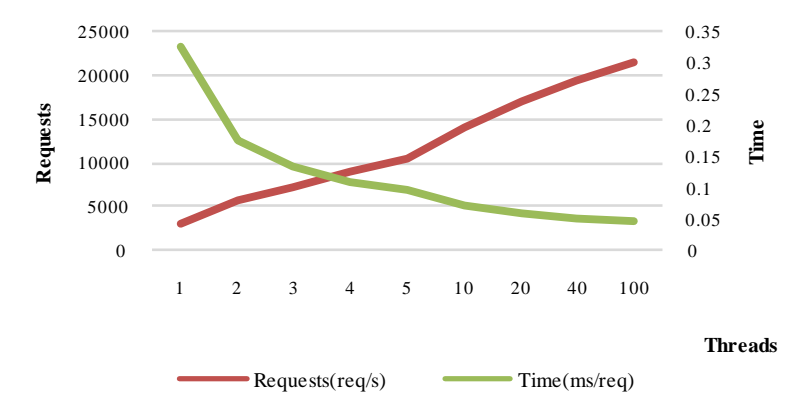

FIGURE IV. COMPUTER STIMULATIONTEST RESULT.

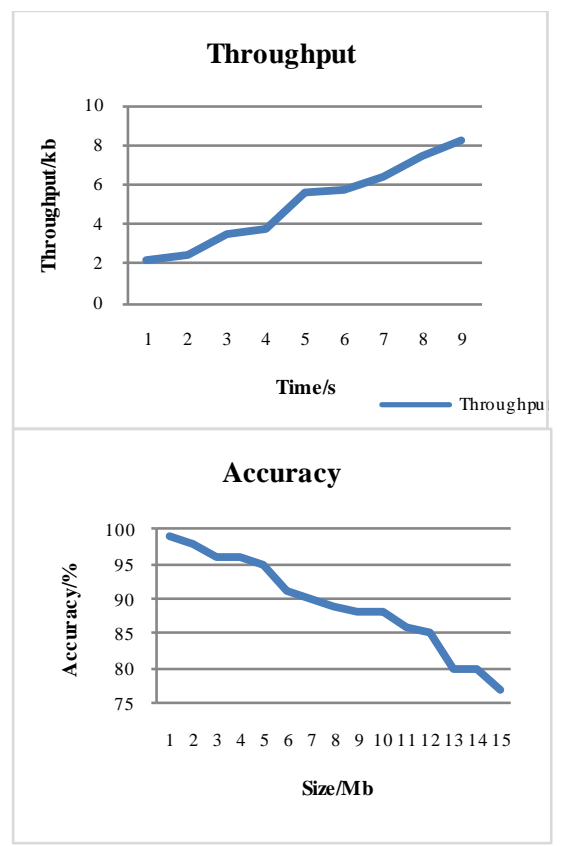

FIGURE V. FIELD TEST RESULT.

\section{CONCLUSION}

At present, researches on teenagers' physical education need the support of real-time data. This paper proposes a design of real-time data acquisition system for physical education. The system insists of wearable data collector, wireless base station, and base station server. Combined with wireless technology and wearable computers, the system realizes real-time data acquisition for multiple users, data preservation and transfer, and other functions. The system offers reliable data for scientific researchers. It also enables P.E. teachers to monitor students' physical exercise in real time, and assists teachers to develop teaching plans. Through computer stimulation test and field test, the availability and performance of the system are proved. In the future, we will deploy this system in real elementary and secondary schools to verify the effectiveness and accuracy of the system.

\section{REFERENCES}

[1] Zhang Jing, Study on Wearable Computer Communication. Chongqing: Chongqing University, pp. 1-2, 2005.

[2] Bass,L, Conveners report of CHI'97 Workshop on Wearable Computers. http://www.bham.ac.uk/ManMechEng/IEG/w1 .html, pp. 11-12, 1992.

[3] Chen Yaqiong, Research on Wireless Network Communication System of Wearable Computing. Beijing: Beijing University of Technology, pp.1-3, 2010.

[4] Wu Yongyi, Intelligent Wearable Device: the next Marketing Strategic Platform. Communications W orld, pp. 30-31, 2014.

[5] A. Pantelopoulos, N.G. Bourbakis, A Survey on Wearable Sensor-Based Systems for Health Monitoring and Prognosis. IEEE Transactions on Systems, Man, and Cybernetics, Part C: Applications and Reviews, Volume 40, Issue 1, pp. 2-3, 2010.

[6] Luo Yafei, Socket Multi-thread Communication based on TCP. Computer Knowledge and Technology, pp. 563-564, 2009.

[7] Sun Feixian, TCP/IP Network Programming Techniques and Examples, Beijing: National Defense Industry Press, pp. 34-36, 2014.

[8] Sarijari, M.A. ; Abdullah, M.S. ; Lo, A. ; Rashid, R.A, Experimental studies of the ZigBee frequency agility mechanism in home area 
networks. 2014 IEEE 39th Conference on Local Computer Networks Workshops (LCN Workshops), pp. 711-712, 2014.

[9] Yongping $\mathrm{Wu}$, Guo Feng, Zhang Meng, The study on coal mine using the Bluetooth wireless transmission. 2014 IEEE Workshop on Electronics, Computer and Applications, pp 101-1018, 2014.

[10] Garber, Lee, Wi-Fi Races into a Faster Future. Computer, Volume 45, Issue 3, pp 13-16, 2012. 